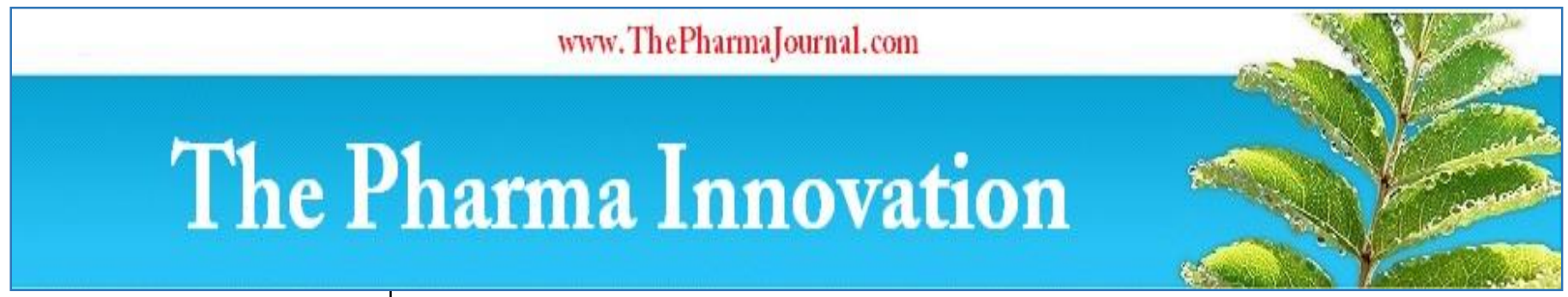

ISSN (E): 2277- 7695

ISSN (P): 2349-8242

NAAS Rating: $\mathbf{5 . 2 3}$

TPI 2021; 10(5): 1315-1321

(C) 2021 TPI

www.thepharmajournal.com

Received: 20-02-2021

Accepted: 14-04-2021

Sudhakar Prakash

Department of Zoology, Shia PG College, Lucknow, Uttar

Pradesh, India

Sadguru Prakash

Department of Zoology, MLKPG

College, Balrampur, Uttar

Pradesh, India

\section{Ethnomedicinal use of fishes by tribal communities in India: A review}

\section{Sudhakar Prakash and Sadguru Prakash}

DOI: $\underline{\text { https://doi.org/10.22271/tpi.2021.v10.i5q.6395 }}$

\begin{abstract}
Study on fish based Zootherapy could be a viable option for discovery of new compounds with therapeutic potentials. In India from primitive time animals are used as medicine to cure different diseases by tribal communities. However, the present generation attitude toward traditional medicine as being unscientific and acculturation are the main causes of decline of such practices in tribal societies. The present review article describes the traditional knowledge of the ichthyotherapy, the use of fishes as medicine to cure human different diseases by tribal communities of India. Respondent listed about 45 fresh water fish species, which are used in primary health care needs of human being. Head, eyes, blood, gall bladder, bile, flesh, fins, bones, mucous, air bladder, otolith etc. are most commonly used parts to treat diseases such as asthma, cough and cold, chicken pox, small pox, kala-azar, diarrhea, malaria, anemia, general weakness, Premenstrual pain, abdominal pain, headache, fever, arthritic etc. The findings are more important for remedial measure and documentation although these have to be tested scientifically and clinically. It is essential to study in detail the use of these fish parts which may in future help medical science and also call for conservation of these fishes. Study on ichthyotherapy could be a viable option for discovery of new compounds with therapeutic potentials. The present ichthyotherapy work has been studied from different published research paper of various authors on ethnozoology/ zootherapeutic studies in India from 1987 to 2020 and it gave us an idea that many types of diseases are cured by fishes and their body parts.
\end{abstract}

Keywords: Ichthyotherapy, ethnomedicine, cure, disease, tribal community

\section{Introduction}

Animals occupy an important position in culture and religion of traditional societies all over the world. Animals, whether domestic or wild, have always been providing a number of goods and services to human beings. The ethnic people maintained a healthy natural environment by making prudent use of the biological resources. The wildlife provides protein and other necessary food materials to the rural tribal communities of developing countries including India. Besides this, animals and animal derived products have always been source of traditional or folk medicine which still prevalent among the tribal communities in India as means of primary health care. Poverty and limited access to modern medicine are the main factors for their dependence on traditional medicine, particularly in rural areas.

World Health Organization (WHO) defines traditional medicine as "the sum total of knowledge, skill and practices based on theories, beliefs and experiences indigenous to different cultures that are used to maintain health as well as to prevent, diagnose, improve or treat physical and mental illnesses."

Traditional medicine is not only a source of healing, but the practice is also an important part of their religion and culture. So, far as modern medicine is concerned, it is reported that more than half of the world's modern drugs are of biological sources and out of 252 that has been selected by WHO as essential to human health, $8.7 \%$ comes from animal sources (Lohani, 2010) ${ }^{[21]}$. Although traditional medicine mainly relies on herbal treatment but animals (whole and their body parts) and their products are also used. It has been reported that more than half of the world's modern drugs are of biological resources.

Due to the vast progress and researches in allopathic drugs there has been a great set-back for the traditional drugs all over the world and in India also. If one looks into the olden days, one can find the frequent use of such traditional drugs by our ancestors to cure the various human ailments. World Health Organization has reported that about 80 percent of the world's populations rely primarily in folk medicine derived from plant and animal origin for immediate cure against illness (Soni et al., 2020) ${ }^{[34]}$.
Corresponding Author: Sadguru Prakash Department of Zoology, MLKPG College, Balrampur, Uttar Pradesh, India 
The traditional medicinal knowledge of the indigenous people across the globe has played an important role in identifying living organisms which are used in treating livestock and human health problems, and loss the knowledge may exert a significance adverse impact on the development of modern medicine (Yirga et al., 2011) ${ }^{[37]}$. Hence, it is important to document, as much as possible, the traditional knowledge of various tribal communities which are on the verge of losing their socio-economic and cultural characteristics (Alves and Rosa, 2007; Jamir and Lal, 2005; Chanu et al., 2014) ${ }^{[1,9,16] .}$

Research interest and activities in the area of ethnobiology and ethnomedicine have increased tremendously since last decade. By the end of the $20^{\text {th }}$ century, the life of the traditional communities was disturbed and disrupted, causing imminent danger of extinction of their culture and ethnomedicinal practices which their forefathers had. In India the traditional knowledge system is fast eroding due to urbanization. So, there is an urgent need to inventories and systematic documentation of such precious ethnobiological knowledge among different ethnic communities before the traditional cultures are completely lost.

Ethnozoology focuses at direct relationship of animals to mankind. Ethnozoology is a branch of biology which deals the relationship with tribal people to animals and their interaction. Ethnozoology is divided into number of branches such as ethnoentomology (deals the therapeutic use of insect in human health care), ethnoichthyology (deals the therapeutic use of fishes in human health care), ethnoherpetology (deals the therapeutic use of snakes in human health care) and ethnoornithology (deals the therapeutic use of birds in human health care). Zootherapy is an integral part of traditional health care practice among the ethnic communities in India but there is a dearth of reports in this regard. A large number of animals had been found providing a number of sobstances with medicinal properties which the people use to treat a wide range of ailments (Chanu et al., 2014) ${ }^{[9]}$. In India there has been only few articles have been published with an ethnozoological approach, although some anthropological, folk and ethnomedicinal studies have focused on the connections between human society and animals (Joseph, 1982; Gupta, 1987; Puspagandhan, 1990; Sharma, 1990; Azmi, 1989 \& 1991; Jamir and Lal, 2005; Azmi and Bharti, 2013; Chanu et al., 2014; Borah and Prasad, 2017; Prakash, 2017; Chhetri et al., 2020; Prakash and Yaday, 2020; Prakash and Verma, 2021) [2, 3, 8-10, 14-16, 18, 27-30].

Fishes have a long history of interaction with humans, thus "ethnoichthyology" is playing an important role in ethnozoological research (Begossi and Garavello, 1990) ${ }^{[7]}$. Besides being a source of food, fish is used for treatment of different ailments by various groups of tribal people around the world and in India also. There are large number of fish species used in traditional medicines, mainly in fishing communities to treat illnesses and health conditions in India (Azmi and Bharti, 2013; Teronpi et al., 2012; Gupta and Day,
2017; Prakash and Yadav, 2020) [3, 14, 34, 29]; however, the documented report on the therapeutic use of fish in health care practices among the tribal population was very scanty and scattered.

India is gifted immense faunal and floral biodiversity, because of the extreme variation in geographical and climatic condition prevailing in the country (Bagde and Jain, 2013) ${ }^{[5]}$. The freshwater fish fauna of India is highly diverse in nature and constituting 1027 species (Gopi et al. 2017) ${ }^{[13]}$. In India, different tribal and ethnic communities are lived throughout the country. People of these communities great knowledge about ichthyofauna and their medicinal value, and they also provide considerable information about the use of freshwater fishes and their by-products as medicine. Most of the rural areas, tribal and ethnic people are totally dependent on local traditional medicinal system for their health care because they are living in remote areas where hospital and other modern medicinal facilities are not available, so they use their traditional knowledge for medicinal purpose and this knowledge is passed through oral communication from generation to generation (Jaroli et al., 2010) ${ }^{[17]}$.

This review paper illustrates the therapeutic use of fish species by Indian ethnic societies. It provides an inventory of the species that have been used for medicinal purposes from ancient times to the present, and analyses the medical use of fishes in the 20th century. Thus, our main objective is to obtain an inventory of the fish species that have been used in India for therapeutic purposes from antiquity to the present. From this we determine which medicinal species have survived to recent times and what diseases or medical conditions they have been used for.

\section{Ethnomedicinal use of some fishes by ethnic communities of India}

Traditional medicine is an indispensable part of primary health-care among the tribal communities in India. Traditional health-care practices of these tribal communities are generally based on religious beliefs and therefore, are an important driver for continuation of their culture (Teronpi et al., 2012) ${ }^{[34]}$. Fishes are the cheapest way for cure of various health disorders. Approximately 45 fresh water fish species are reported to use in the treatment of about 45 disease conditions as traditional medicine by different tribal communities in various parts of India. The inventoried fresh water fish species belongs to 9 order: Cypriniformes (20), Siluriformes (9), Ophiocephaliformes (5), Synbranchiformes (3), Perciformes (2), Anabantiformes (2), Clupiformes (2), Anguilliformes (1) and Beloniformes (1). Traditional health care practices among the tribal population in India include oral therapy, contact therapy, by preparing solutions or powders. The order, scientific name, common name and conservation status of fishes used by ethnic communities in India for medicinal purpose along with method of application is given in the table.

Table 1: List of freshwater Ichthyofauna uses as medicinal purposes by tribal communities in different part of India

\begin{tabular}{|c|c|c|c|c|}
\hline $\begin{array}{c}\text { Scientific/Common name } \\
\text { (Conservation status) }\end{array}$ & Parts used & Disease condition & Method of application/consumption & $\begin{array}{l}\text { Reference } \\
\text { No. }\end{array}$ \\
\hline \multicolumn{5}{|c|}{ Order: Clupiformes } \\
\hline $\begin{array}{c}\text { Notopterus notopterus } \\
\text { (Pallas)/Featherback (LC) }\end{array}$ & $\begin{array}{l}\text { Whole } \\
\text { body }\end{array}$ & Abdominal \& Delivery Pain & Burned and Cooked with black Pepper & [14] \\
\hline $\begin{array}{c}\text { Hilisa ilisha } \\
\text { (Hamiliton)/herring (LC) }\end{array}$ & Body oil & $\begin{array}{l}\text { Arthritis, Cracked heels, } \\
\text { Nightblindness, Scurvy }\end{array}$ & Used as ointment for hands, heels and legs & [9] \\
\hline
\end{tabular}




\begin{tabular}{|c|c|c|c|c|}
\hline \multirow{8}{*}{$\begin{array}{l}\text { Labeo rohita } \\
\text { (Hamiliton)/Rohu, carp } \\
\text { (LC) }\end{array}$} & $\begin{array}{l}\text { Whole } \\
\text { body }\end{array}$ & $\begin{array}{l}\text { Rheumatism, Galactoschesia, } \\
\text { Whooping cough, Bronchitis, } \\
\text { Loss of sexual vigour }\end{array}$ & Fish Curry & {$[14,29,31]$} \\
\hline & Fat & Facial Paralysis & $\begin{array}{l}\text { Warmed, massaged externally thrice daily, for three } \\
\text { weeks. }\end{array}$ & [29] \\
\hline & Eye, Oil/Fat & Night blindness & $\begin{array}{c}\text { The boiled decocation of eye and fish oil is given } 2 / 3 \\
\text { times in a week }\end{array}$ & [9] \\
\hline & Liver & Night blindness & $\begin{array}{l}\text { Boiled in water, soup drunk and remainder cooked } \\
\text { and eaten }\end{array}$ & \multirow[b]{2}{*}{ [29] } \\
\hline & $\begin{array}{l}\text { Scales/ } \\
\text { Teeth/ } \\
\text { Bones }\end{array}$ & Weak-sight, Eye troubles & $\begin{array}{c}\text { Incinerated powdered mixed in honey and applied into } \\
\text { the eyes at bed time. }\end{array}$ & \\
\hline & Stone & Kidney stone & $2 \mathrm{mg}$ stone taken orally & [5] \\
\hline & $\begin{array}{l}\text { Gall } \\
\text { Bladder } \\
\text { /Bile }\end{array}$ & $\begin{array}{l}\text { Gastric enteritis, Gastric ulcer, } \\
\text { Intestinal cancer }\end{array}$ & $\begin{array}{c}\text { Ground the gall bladder with water or 8-10 drops bile } \\
\text { diluted in one glass of water and drunk in empty } \\
\text { stomach }\end{array}$ & {$[17,29]$} \\
\hline & $\begin{array}{l}\text { Cervical } \\
\text { vertebra }\end{array}$ & Urine blockage Problem & $\begin{array}{c}\text { A cervical vertebra is rubbed with water and this } \\
\text { essence water is taken }\end{array}$ & [25] \\
\hline \multirow{3}{*}{$\begin{array}{l}\text { Labeo pangusia } \\
\text { (Hamiliton)/Carp fish (LC) }\end{array}$} & \multirow[t]{2}{*}{ Flesh } & $\begin{array}{l}\text { Food Poisoning Brain } \\
\text { improvement }\end{array}$ & $\begin{array}{l}\text { The cooked fish is given as antidote for food } \\
\text { poisoning }\end{array}$ & [9] \\
\hline & & Weakness after delivery & Boiled fish is taken as tonic & \multirow{2}{*}[17,34]{} \\
\hline & Bile & Stomach ache & Bile is taken orally & \\
\hline $\begin{array}{c}\text { Labeo gonius } \\
\text { (Hamiliton) /Carp fish } \\
\text { (LC) }\end{array}$ & $\begin{array}{l}\text { Flesh, } \\
\text { Bone }\end{array}$ & Obesity; Allergy & & {$[11,26,31]$} \\
\hline $\begin{array}{c}\text { Catla catla } \\
\text { (Hamiliton)/BCatla (LC) }\end{array}$ & Opercul-um & Ripenining of boils & $\begin{array}{l}\text { Crushed operculum is made into paste and applied to } \\
\text { affected area }\end{array}$ & [9] \\
\hline $\begin{array}{c}\text { Barbus sp. } \\
\text { (Cuvier \& Cloquet)/Ray } \\
\text { fined fish (LC) } \\
\end{array}$ & $\begin{array}{l}\text { Slime/ } \\
\text { Mucous }\end{array}$ & Chicken pox & Mucous applied on affected parts & [11] \\
\hline \multirow{6}{*}{$\begin{array}{l}\text { Puntius sp. } \\
\text { (Hamiliton) } \\
\quad \text { (LC) }\end{array}$} & \multirow{4}{*}{$\begin{array}{l}\text { Whole } \\
\text { body }\end{array}$} & Eye problem & Cooked with black pepper & {$[12]$} \\
\hline & & Blood Purifer & Cooked with bamboo shoot & {$[14,34]$} \\
\hline & & Common cold & Cooked with chili & \\
\hline & & Plague, Ulcer (gastric) & $\begin{array}{l}\text { Fermented fish is crushed into a paste with cooked } \\
\text { rice; the paste is taken before meal for one week }\end{array}$ & [9] \\
\hline & \multirow{2}{*}{ Head } & Night blindness & Cooked head consumed & \multirow{2}{*}{ [34] } \\
\hline & & Memory & Boiled head consumed & \\
\hline $\begin{array}{l}\text { Amblypharyngodon mola } \\
\text { (Hamiliton)/Mola (LC) }\end{array}$ & $\begin{array}{l}\text { Whole } \\
\text { body }\end{array}$ & $\begin{array}{c}\text { Premenstrual pain; } \\
\text { Chicken \& small pox, Pain, } \\
\text { Asthma } \\
\end{array}$ & $\begin{array}{l}\text { Boiled or Cooked the Fish with light spices and } \\
\text { consumed }\end{array}$ & {$[11,14,8,29]$} \\
\hline \multirow{2}{*}{$\begin{array}{l}\text { Semiplotus sp. } \\
\text { (Bleeker)/king fish (LC) }\end{array}$} & $\begin{array}{l}\text { Stomach } \\
\& \text { Gut }\end{array}$ & $\begin{array}{c}\text { Stomach ache } \& \text { digestive } \\
\text { problems }\end{array}$ & \multirow{2}{*}{ Cooked/ boiled with black pepper and salt } & \multirow{2}{*}{ [11] } \\
\hline & $\begin{array}{l}\text { Whole } \\
\text { body }\end{array}$ & Small Pox & & \\
\hline $\begin{array}{l}\text { Chela bacaila (Hamilton) } \\
\text { /Chilwa }\end{array}$ & $\begin{array}{l}\text { Whole } \\
\text { body }\end{array}$ & $\begin{array}{l}\text { Weal-sight, Night blindness, } \\
\text { Eye ailments }\end{array}$ & $\begin{array}{l}\text { Boiled, squashed, macerated in water and taken; } \\
\text { Deep fried in mustard oil, extracted the oil from the } \\
\text { body and applied into eyes. }\end{array}$ & [29] \\
\hline $\begin{array}{c}\begin{array}{c}\text { Cyprinus carpio } \\
\text { (Linnaeus) }\end{array} \\
\text { /Common carp (VU/TH) }\end{array}$ & Bile, Fat & Fever, Headache & Bile are swallowed; Fat is taken/eaten & [20] \\
\hline $\begin{array}{c}\text { Danio aequipinnatus } \\
\text { (McClelland)/Giant danio } \\
\text { (LC) } \\
\end{array}$ & $\begin{array}{l}\text { Whole } \\
\text { body }\end{array}$ & Constant spitting & Boiled fish is consumed & [34] \\
\hline $\begin{array}{c}\text { Tor puntitora } \\
\text { (Hamiliton)/Mahaseer (EN) }\end{array}$ & Bile & Asthma & Fresh bile taken orally & [22] \\
\hline $\begin{array}{l}\text { Tor tor }(\text { Hamiliton }) / \\
\text { Mahaseer }(\mathrm{NT})\end{array}$ & Bile & High fever & Fresh bile taken orally & [21] \\
\hline $\begin{array}{c}\text { Psilorhynchus } \\
\text { pseudechenesis (Menon \& } \\
\text { Dutta)/ Nepalese Minnow } \\
\text { (LC) } \\
\end{array}$ & $\begin{array}{l}\text { Whole } \\
\text { body, Bile }\end{array}$ & $\begin{array}{l}\text { High fever, problem in Urine } \\
\text { discharge }\end{array}$ & $\begin{array}{l}\text { Cooked and eaten orally. } \\
\text { Bile is taken orally in case of high fever }\end{array}$ & [21] \\
\hline $\begin{array}{c}\text { Psilorhynchus balitora } \\
\text { (Hamiliton)/Balitora } \\
\text { minnow (LC) }\end{array}$ & $\begin{array}{l}\text { Whole } \\
\text { body }\end{array}$ & Diarrhoea & & [11] \\
\hline \multirow[t]{2}{*}{$\begin{array}{l}\text { Schizothorax plagiastomus } \\
\text { (Heckel)/Snow trout }\end{array}$} & $\begin{array}{l}\text { Gastro- } \\
\text { intestinal } \\
\text { Tract } \\
\end{array}$ & $\begin{array}{l}\text { Gastritis and } \\
\text { loss of appetite }\end{array}$ & $\begin{array}{l}\text { Gastrointestinal tract along with its contents is boiled } \\
\text { in water and eaten. }\end{array}$ & \multirow[t]{2}{*}[21,22]{} \\
\hline & Bile & Body ache, Headache & Bile is taken orally & \\
\hline Barilius bendelisis & Whole body & Constipation, deworming & Cooked with pieces of Pinus insularis \& Murdania & {$[9]$} \\
\hline
\end{tabular}




\begin{tabular}{|c|c|c|c|c|}
\hline (Hamiliton) (LC) & & & \begin{tabular}{|c|} 
nudiflora and water without oil \\
\end{tabular} & \\
\hline $\begin{array}{l}\text { Osteobrama belangeri } \\
\text { (Valenciennes) (NT) }\end{array}$ & Fat/Oil & $\begin{array}{l}\text { Aphrodisiac and } \\
\text { Loosening of vaginal muscles }\end{array}$ & $\begin{array}{l}\text { Oil is extracted from pectoral muscles and mixed with } \\
\text { root juice of Musa paradisiacal atd taken twice a day } \\
\text { for one month }\end{array}$ & [9] \\
\hline $\begin{array}{l}\text { Osteobrama Cotio cotio } \\
\text { (Hamiliton) (LC) }\end{array}$ & Whole body & Ringworm & $\begin{array}{c}\text { Sundried whole body crushed into a powder and used } \\
\text { as dried powder }\end{array}$ & [9] \\
\hline $\begin{array}{c}\text { Esomus danricus } \\
\text { (Hamiliton)/Flying barb } \\
\text { (LC) }\end{array}$ & Whole body & Lactation & $\begin{array}{l}\text { Smoked fish cooked with Allium sativum and Allium } \\
\text { cepa as curry and takes twice daily for two days }\end{array}$ & [9] \\
\hline \multicolumn{5}{|c|}{ Order: Siluriformes } \\
\hline \multirow{4}{*}{$\begin{array}{l}\text { Heteropneutes fossilis } \\
\text { (Bloch)/Singhi (LC) }\end{array}$} & \multirow{3}{*}{$\begin{array}{l}\text { Whole } \\
\text { body }\end{array}$} & Anemia' Weakness after delivery & Boiled Fish & {$[14,34]$} \\
\hline & & Pain, Wound healing, Impotency & flesh cooked with black pepper than consumed & {$[8,29]$} \\
\hline & & Blood Purifier, Anemia & Cooked/Boiled with Phylogacanthus thysiflorus & [9] \\
\hline & Brain & Sting by the fish itself & $\begin{array}{l}\text { Raw brain is consumed when stung by fish, as } \\
\text { analgesia }\end{array}$ & [34] \\
\hline \multirow{5}{*}{$\begin{array}{c}\text { Clarias batrachus } \\
\text { (Linnaeus)/Mangur (LC) }\end{array}$} & $\begin{array}{c}\text { Flesh } \\
\text { (Whole body } \\
\text { except skin) }\end{array}$ & Anemia, Malnutrition & $\begin{array}{l}\text { Boiled with Emblica officinalis and Allium sativum } \\
\text { and make as soup }\end{array}$ & [9] \\
\hline & \multirow{4}{*}{$\begin{array}{l}\text { Whole } \\
\text { body }\end{array}$} & Small pox & Cooked fish & \multirow{2}{*}{$11,14,34$} \\
\hline & & Weakness after delivery & Boiled fish is taken regularly to regain the strength & \\
\hline & & $\begin{array}{l}\text { Body ache, Bronchitis Wound } \\
\text { healing }\end{array}$ & Cooked with black pepper and take orally & \multirow{2}{*}[8,29]{} \\
\hline & & Gum-boils, Teething problem & $\begin{array}{l}\text { Incinerated powder mixed with honey and applied on } \\
\text { jaws of babies }\end{array}$ & \\
\hline \multirow{3}{*}{$\begin{array}{l}\text { Mystus sp. (Bloch) } \\
\text { /Tengara (LC) }\end{array}$} & \multirow{3}{*}{$\begin{array}{l}\text { Whole } \\
\text { body }\end{array}$} & Small pox & \begin{tabular}{|c|} 
Cooked fish \\
\end{tabular} & {$[14,34]$} \\
\hline & & After delivery to new mother & Used as tonic & [11] \\
\hline & & Dysentery & $\begin{array}{c}\text { Boiled with Portulaca oleracea, curry taken two times } \\
\text { daily for three days }\end{array}$ & [9] \\
\hline Aorichthys seenghala & Liver, Eye & Nightblindness & \begin{tabular}{|c|} 
Boiled liver and eyeball \\
\end{tabular} & [9] \\
\hline (Sykes) & Bile & Chronic Fever & Fresh bile with water & \\
\hline \multirow{6}{*}{$\begin{array}{l}\text { Wallago attu } \\
\text { (Schneider)/Pardhin (LC) }\end{array}$} & Head & Liver tonic & Boiled head taken regularly to regain the strength & {$[14,34]$} \\
\hline & Barbeles & Diarrhoea; Impotency & $\begin{array}{c}\text { Rosted barbell is mixed with powdered, tender leaves } \\
\text { of Psidium guajawa }\end{array}$ & [9] \\
\hline & $\begin{array}{l}\text { Air } \\
\text { bladder }\end{array}$ & Asthma & Used in diet & [26] \\
\hline & Flesh & $\begin{array}{l}\text { Asthma, Breathing problem } \\
\text { Tuberculosis }\end{array}$ & Cooked with light spices and taken orally. & \multirow{3}{*}{ [29] } \\
\hline & \multirow[t]{2}{*}{ Fat } & Loss of erectile power & $\begin{array}{c}\text { Warm fat massaged on the lumbo-sacral region at } \\
\text { night }\end{array}$ & \\
\hline & & Burn, Wound, Foot cracks & Warmed and applied externally & \\
\hline $\begin{array}{l}\text { Chaca chaca } \\
\text { (Hamiliton)/Devil fish } \\
\text { (LC) }\end{array}$ & $\begin{array}{l}\text { Whole } \\
\text { body }\end{array}$ & Polio & $\begin{array}{c}\text { Dried fish is boiled or cooked with spice and taken } \\
\text { orally }\end{array}$ & {$[11,14]$} \\
\hline \multirow{2}{*}{$\begin{array}{l}\text { Bagarius bagarius } \\
\text { (Hamilton)/Gangetic } \\
\text { goonch }(\mathrm{NT})\end{array}$} & Fat & Gout, Rheumatism \& Joint pain & Massaged on the joints & {$[20]$} \\
\hline & $\begin{array}{r}\text { Fins \& } \\
\text { Bones }\end{array}$ & Body Burn; Stomach Pain & 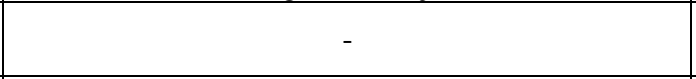 & [11] \\
\hline Amblyceps sp. (Blyth) & Bones & Body Burn & & [11] \\
\hline $\begin{array}{l}\text { Eutropiichthys vacha } \\
\text { (Hamiliton) }(\mathrm{EN})\end{array}$ & Flesh & Tuberculosis, To improve Brain & Used as curry two times in a week for one month & [9] \\
\hline \multicolumn{5}{|c|}{ Order: Ophiocephaliformes } \\
\hline \multirow{7}{*}{$\begin{array}{c}\text { Channa Punctatus } \\
\text { (Bloch)/Snake headed fish } \\
\text { (LC) }\end{array}$} & Eyes & Corn or Clavus & $\begin{array}{c}\text { Eyes mixed with salt and applied to affected part to } \\
\text { remove corn }\end{array}$ & {$[14,34]$} \\
\hline & Head & Swelling of the testicles & $\begin{array}{l}\text { Heads are tapped on the affected testicle to control } \\
\text { swelling }\end{array}$ & \multirow[t]{2}{*}{ [34] } \\
\hline & Bile & Malaria & Bile is taken orally twice a day till recovery & \\
\hline & \multirow{3}{*}{$\begin{array}{l}\text { Whole } \\
\text { body }\end{array}$} & $\begin{array}{l}\text { After delivery to new mother; } \\
\text { Body Pain }\end{array}$ & Used as tonic & [11] \\
\hline & & Diarrhea & Boiled fish is consumed & [34] \\
\hline & & Eye-ailments & Oil extract applied on the eyes & \multirow[t]{2}{*}{ [29] } \\
\hline & Flesh & General weakness, Tuberculosis & Prepare soup with light spices and taken & \\
\hline \multirow{2}{*}{$\begin{array}{l}\text { Channa striatus (Bloch) } \\
\text { /Snake headed fish (LC) }\end{array}$} & \multirow{2}{*}{$\begin{array}{l}\text { Whole } \\
\text { body }\end{array}$} & Diabetes, Pain, High BP & Boiled in water with salt and make a soup than drink & [29] \\
\hline & & Vitamins and general body tonic & Boiled with Clarias batrachus as soup & [9] \\
\hline $\begin{array}{l}\text { Channa orientalis }(\text { Bloch }) / \\
\text { Smooth breasted snake fish } \\
\text { (VU) }\end{array}$ & Whole body & $\begin{array}{l}\text { Stone case, opening of swelling/ } \\
\text { boil inside the body }\end{array}$ & Cooked with Cissus adnata as curry once a week & [9] \\
\hline $\begin{array}{c}\text { Channa stevvartii (Playfair) } \\
\text { /Snake headed fish (LC) }\end{array}$ & $\begin{array}{l}\text { Whole } \\
\text { body }\end{array}$ & Diabetes, Pain, High BP & Boiled in water and consumed & [8] \\
\hline \multirow{2}{*}{$\begin{array}{c}\text { Channa gachua (Hamiliton) } \\
\text { /Snake headed fish (LC) }\end{array}$} & \multirow{2}{*}{$\begin{array}{l}\text { Whole } \\
\text { body }\end{array}$} & Abdominal pain & Boiled fish & {$[11,14]$} \\
\hline & & Dysentery & Fish is mixed with salt and warped in banana leaf, & {$[34]$} \\
\hline
\end{tabular}




\begin{tabular}{|c|c|c|c|c|}
\hline & & & boiled and eatern & \\
\hline & & Gall bladder stone & Flesh is cooked and consumed & [12] \\
\hline & & Asthma, Tuberculosis & Dried powder mixed with honey and consumed & \multirow{3}{*}{ [29] } \\
\hline & Flesh & Diabetes General debility & $\begin{array}{l}\text { Prepare soup with salt and light black pepper than } \\
\text { taken orally. }\end{array}$ & \\
\hline & Mucous & Menstrual irregularity & $\begin{array}{c}\text { Dermal secretions are scrapped, dried and transformed } \\
\text { into pills and taken orally }\end{array}$ & \\
\hline \multicolumn{5}{|c|}{ Order: Anabantiformes } \\
\hline $\begin{array}{l}\text { Colisa sota (Hamiliton) } \\
\text { (LC) }\end{array}$ & Whole body & Post delivery Period & $\begin{array}{l}\text { Sundried fish is crushed with Alocassia indica and } \\
\text { made into a fermented paste }\end{array}$ & [9] \\
\hline \multirow{2}{*}{$\begin{array}{c}\text { Anabas testudineus } \\
\text { (Bloch)/Climbing perch } \\
\text { (LC) }\end{array}$} & \multirow{2}{*}{$\begin{array}{l}\text { Whole } \\
\text { body }\end{array}$} & Dymennorhea & Head boiled with spice & [14] \\
\hline & & General weakness, Malaria & Boiled fish is taken to regain the strength & [11] \\
\hline \multicolumn{5}{|c|}{ Order: Anguilliformes } \\
\hline \multirow{5}{*}{$\begin{array}{c}\text { Anguilla bengalensis } \\
\text { (Gray)/Fresh water Eel } \\
\text { (NT) }\end{array}$} & Whole Body & Piles \& Meningitis & Cooked with Stelloria media and taken as soup & [9] \\
\hline & Fats & Rheumatoid arthritis & Fat is applied and message to relieve pain & {$[14,34]$} \\
\hline & $\begin{array}{l}\text { Body } \\
\text { mucous }\end{array}$ & Burns & Mucous applied on the affected parts & [11] \\
\hline & Blood & Weakness & $\begin{array}{l}\text { Blood of eel mixed with turmeric (hot dry) and taken } \\
\text { orally }\end{array}$ & [26] \\
\hline & $\begin{array}{l}\text { Dried Tail } \\
\text { And Dried } \\
\text { gall bladder }\end{array}$ & For easy and normal delivery & $\begin{array}{c}\text { Dried tail of the fish is soaked in water and the } \\
\text { decanted water is given to the women at time of her } \\
\text { delivery. } \\
\text { Dried gall bladder is soaked in water and rubbed on } \\
\text { the stone to obtain its smooth paste. } \\
\text { It is believed that it facilitates easy expulsion of } \\
\text { placenta. }\end{array}$ & [22] \\
\hline \multicolumn{5}{|c|}{ Order: Synbranchiformes } \\
\hline \multirow{4}{*}{$\begin{array}{c}\text { Amphipnous cuchia } \\
\text { (Hamiliton)/Mud Eel (LC) } \\
\text { Or } \\
\text { Monopterus cuchia } \\
\text { (Cuchia Eel) }\end{array}$} & \multirow[b]{2}{*}{ Meat } & Premenstrual abdominal pain & Boiled fish & \multirow[b]{2}{*}[11,14,34]{} \\
\hline & & $\begin{array}{c}\text { Kala-azar, Weakness after } \\
\text { delivery }\end{array}$ & Raw fish is boiled and taken orally & \\
\hline & Blood & $\begin{array}{l}\text { Asthma, Jaudice, Anemia, Kala- } \\
\text { azar, Weakness, Diabetes }\end{array}$ & Raw Blood is Consumed & {$[11,14,24,29,34$} \\
\hline & $\begin{array}{c}\text { Gall } \\
\text { bladder }\end{array}$ & $\begin{array}{l}\text { Asthma, Rhinitic (Inflamation in } \\
\text { nasal lining by sneezing \& runny } \\
\text { nose) }\end{array}$ & $\begin{array}{l}\text { Prepare a paste from Fresh or sun dried Gall bladder } \\
\text { and taken orally }\end{array}$ & [22] \\
\hline $\begin{array}{c}\text { Monopterus albus } \\
\text { (Zuiew)/Asian swamp eel } \\
\text { (LC) }\end{array}$ & Flesh & $\begin{array}{l}\text { Undernurished and anemic child } \\
\text { and adult }\end{array}$ & $\begin{array}{l}\text { The flesh is cooked in fresh milk and curry is taken } \\
\text { once a week }\end{array}$ & [9] \\
\hline \multirow{2}{*}{$\begin{array}{c}\text { Mastacembelus armatus } \\
\text { (Lacepede) } \\
\text { /Zig-Zag eel; Spiny eel (LC) }\end{array}$} & $\begin{array}{l}\text { Whole } \\
\text { body }\end{array}$ & Carbuncle & & [11] \\
\hline & $\begin{array}{l}\text { Flesh, Liver, } \\
\text { Bile }\end{array}$ & $\begin{array}{l}\text { Kwashiorkor, Night blindness, } \\
\text { Chronic Fever }\end{array}$ & $\begin{array}{c}\text { Liver is boiled and bile is crushed with water. Soup is } \\
\text { taken two times a day for one week. }\end{array}$ & [9] \\
\hline \multicolumn{5}{|c|}{ Order: Beloniformes } \\
\hline $\begin{array}{c}\text { Xenetodon cancila } \\
\text { (Hamilton)/Gar fish (LC) }\end{array}$ & $\begin{array}{l}\text { Whole } \\
\text { body }\end{array}$ & Joint Pain, Swelling & $\begin{array}{c}\text { Cooked and consumed; Spines and bones is used to } \\
\text { pick out the clotted blood }\end{array}$ & {$[11,14]$} \\
\hline \multicolumn{5}{|c|}{ Order: Perciformes/Gobiiformes } \\
\hline $\begin{array}{c}\text { Glossogobius gutum } \\
\text { (Hamilton)/Tank goby (LC) }\end{array}$ & Flesh & $\begin{array}{c}\text { Nocturnal enuresis (involuntary } \\
\text { urination during sleep) }\end{array}$ & Cooked and eaten & [26] \\
\hline $\begin{array}{c}\text { Pseudosciaena coitor } \\
\text { (Hamilton) }\end{array}$ & Internal ear & $\begin{array}{c}\text { Rickets in children and trouble in } \\
\text { urinary passage. }\end{array}$ & $\begin{array}{l}\text { Otoliths, hard calcium carbonate bodies present in } \\
\text { internal ear are taken orally }\end{array}$ & {$[15]$} \\
\hline
\end{tabular}

Pisces are one of the widely used animal groups by the ethnic communities of the India. Different ethnic groups are using 45 different species of fresh water fishes for medicinal purpose. The whole body and their body parts like head, eyes, blood, gall bladder, bile, flesh, fins, bones, mucous, air bladder, otolith etc. are most commonly used to cure diseases such as asthma, cough and cold, chicken pox, small pox, kala-azar, diarrhea, malaria, anemia, general weakness, Premenstrual pain, abdominal pain, headache, fever, arthritic etc. Mostly treated ailments are the asthma, anemia, joint pain, burn, anaemia, etc. Labeo sp., Puntius Sp., Channa sp., Anguilla sp., Heteropneustes fossilis, Clarias batrachus, Wallago attu, and Amphipnous cuchia are commonly used in treating number of human ailments. The population of these medicinal important fishes was drastically declined in their natural habitats due to indiscriminate and uncontrolled fishing and habitat destruction especially by using chemicals (Chanu et al., 2014) ${ }^{[9]}$.

\section{Conclusion}

Traditional medicine is based on resource availability and therefore, study of such practices provides information about diversity and distribution of organisms in the past. Further, traditional knowledge of indigenous/tribal people can provide leads for sustainable use and management of natural biological resources. Today however, overexploitation of biological resources has pushed our natural resources toward the brink of collapse. Besides being a source of food, fish is 
used in health-care practices among the tribal population of India. Poverty and limited access to modern medicine are the main factors for their dependence on traditional medicine, particularly in rural areas.

From this review, it can be concluded that people belonging to the different ethnic communities on India have a rich Ethnozoological knowledge and biological resources. Number of fishes had been found providing a number of substances with medicinal properties which the people use to treat a wide range of ailments. The present study indicated that fresh water fishes are still being used by different tribal communities of India, to treat various illnesses. The review throws light on the fact that fishes can be used in treating a number of human ailments. The empirical knowledge reported in this study will provide outstanding possibilities for the discovery of new sources of medicine for the drug industry. If fish can be used as a good source of simple forms of medicine traditionally used earlier, the common people may have an easy way to avoid the high cost of modern medical treatments, thereby improving their economic condition. Industrial pollution and destructive fishing practices by poisoning water bodies with synthetic chemicals pose serious threats to aquatic fauna especially freshwater fishes. Detailed investigations and proper traditional management strategy is urgently required to keep each species intact before the population of species dwindle (Chanu et al., 2014) ${ }^{[9]}$. I hope that this review will be helpful in further research in the field of ethnozoology, zootherapy, ethnopharmacology and biodiversity conservation point of view.

\section{Acknowledgement}

Authors acknowledge the immense help received from the scholars whose articles are cited and included in references of this manuscript. The authors are also grateful to authors/editors/publishers of all those article, journals and books from where the literature for this article has been reviewed and discussed.

\section{References}

1. Alves RRN, Rosa IL. Zootherapeutic practices among fishing communities in North and Northeast Brazil: a comparison. J Ethnopharmacol 2007;111:82-103.

2. Azmi HK. Ethnozoology: Some medicinal aspects Part 1: Drugs of mammalian Origin used by certain tribes in Rajasthan. J Vety. Physol. Alld. Sci 1989;8:19-35.

3. Azmi HK. Use of homoeothermic animals as traditional drugs in certain tribes of eastern (Purvanchal) Uttar Pradesh. Uttar Pradesh Journal of Zoology 1991;11(1):36-42.

4. Azmi HK, Bharti. Use of animal facts as drugs by the tribals of Malwa region of Madhya Pradesh. Indian J L. Sci 2013;3(1):113-116.

5. Bagde N, Jain S. An ethnozoological studies and medicinal values of vertebrate origin in the adjoining areas of Pench National Park of Chhindwara District of Madhya Pradesh, India. International Journal of Life Sciences 2013;1(4):278-283.

6. Bagde N, Jain S. Traditional and Ethnozoological Practices by Tribes and Rurals of Chhindwara District of Madhya Pradesh, India. World Journal of Pharmaceutical and Medical Research 2017;3(8):263-268.

7. Begossi A, Garavello JC. Notes on ethnoichthyology of fisherman from the Tocantins River. Acta Amazonica 1990;20:341-352.
8. Borah MP, Prasad SB. Ethnozoological study of animals based medicine used by traditional healers and indigenous inhabitants in the adjoining area of gibbon wildlife Sanctuary, Assam, India. Journal of Ethnobiology and Ethinomedicine 2017;13:39. DOI 10.1186/s13002-017-0167-6

9. Chanu TA, Teron R, Singh SK. Ethnomedicinal use of certain fish species by ethnic groups of Bishnupur District in Manipur, NE India. World Journal of Environmental Biosciences 2014;5(2):1-5.

10. Chhetri S, Bhutia D, Yonle R, Guruhg Y. Ethnozoological practices among the inhabitants of Darjeeling hills of West Bengal, India. Uttar Pradesh Journal of Zoology 2020;41(14):9-18.

11. Das K, Choudhury S, Chanreila K, Nonglait L. Zootherapy among the Ethnic groups of North Eastern region of India- A Critical Review. Journal of Critical Reviews 2017;4(2):1-9.

12. Gogoi C, Bora M. Zoo-therapeutic practices among the deori tribes of Dhemaji district, Assam, India. International Journal of Fauna and Biological Studies 2020;7(4):196-198

13. Gopi KC, Mishra SS, Kosygin L. Pisces. Chapter 33, pp. 527-570. In: Chandra K, Gopi KC, Rao DV, Valarma-thi $\mathrm{K}$ and Alfred JRB (Eds) Current Status of Freshwater Faunal Diversity in India. Director, Zoological Survey of India, Kolkata, India 2017, 624.

14. Gupta T, Dey M. Ichthyotherapy: use of fishes as medicine by ethnic Karbi people of Assam, India. European Journal of Pharmaceutical and Medical Research 2017;4(10):341-343.

15. Gupta SK. On the fish fauna of Banda district VIIEthnozoological notes of Pseudosciaena coitor (Hamilton). Proc. Nat. Acad. Sci. India 1987;57(B)II:199-200.

16. Jamir NS, Lal P. Ethnozoological practices among Naga tribes, Indian Journal of Traditional Knowledge 2005;4(1):100-104.

17. Jaroli DP, Mahawar MM, Vyas N. An ethnozoogical study in the adjoining areas of Mount Abu wildlife Sanctuary, India, Journal of Ethnobiology and Ethnomedicine 2010;6(6):1-8.

18. Joseph ANT. Use of animals and drugs in in certain tribals of Madhya Pradesh. Proc. Asian Cong. On Traditional Asian Medicines, Bombay 1982.

19. Kamble VS. Etnozoological Survery of Nomadic Tribs from Drought Prone Region Sangola, Dist. Solapur (Ms) India. World Journal of Pharmaceutical Research 2014;3(9):780-787.

20. Lalramnghinglova $\mathrm{H}$. Ethnobiology in Mizoram state: Folklore Medicozoology. Bull. Ind. Inst. Hist. Med 1999;29;123-148.

21. Lohani Usha. Man-animal relationships in Central Nepal. Journal of Ethnobiology and Ethnomedicine 2010;6:31. Doi.10.1186/1746-4269-6-31

22. Lohani Usha. Zootherapeutic knowledge of two ethnic populations from central Nepal. Ethno. Med 2012;6(14):45-53.

23. Mahawar MM, Jaroli DP. Traditional Zootherapeutic studies in India: a review. Journal of Ethnobiology and Ethnomedicine 2008;4:17. doi: 10.1186/1746-4269-4-17.

24. Misar SD, Subhas M, Khinchi PJ, Mohitkar SP. Ethnozoological studies among adjoining areas of Pandharkawda Taluka of Yavatmal District, Maharashtra, 
India. International Journal of Researches in Biosciences, Agriculture and Technology. 2016, 97-100.

25. Pandey A. Use of Animal as Traditional Medicine in India. IOSR Journal of Environmental Science, Toxicology and Food Technology 2015;1(3):48-52.

26. Paul S. Ethnozoological knowledge among Mising Tribes of Dhemaji, Assam. International Journal of Engineering, Science and Mathematics 2018;7(3):53-65.

27. Prakash S. Medico-Ethnozoological studies on homoeothermic vertebrates of devipatan division of Uttar Pradesh, India. International Journal of Fauna and Biological Studies 2017;4(6):62-66.

28. Prakash S, Verma AK. Relevance of Ethnomedicines of Invertebrate origin used by Tribals at Indo-Nepal Border. International Research Journal of Biological Sciences 2021;10(1):39-39.

29. Prakash S, Yadav DK. Medico-ethnozoological studies on anamniotes fauna of Devipatan division of Uttar Pradesh, India. International Journal of Zoology and Applied Biosciences 2020;5(5):222-227.

30. Puspagandhan P. Animals and animal products used in local health traditions of India, Nagarjun 1990;33:189.

31. Puspagandhan P, George V, Sreedevi P, Tjinu TP, Ninawe A. Ethnozoological knowledge of India Scheduled tribe, Scheduled caste and rural communities. Indian Journal of Traditional Knowledge 2014;13(4):735741.

32. Sharma VP. Ethnozoological studies on the invertebrates of Rajasthan State, India, Uttar Pradesh J Zool 1990;10(2):133.

33. Silvano RAM, Ramires M, Zuanon J. Effects of fisheries management on fish communities in the floodplain lakes of a Brazilian Amazonian. Reserv. Ecol. Freshw Fish 2009;18:156-166.

34. Soni C, Dawa B, Rujas Y, Yuvraj G. Ethnozoological practices among the inhabitants of Darjeeling hills of West Bengal. Uttar Pradesh Journal of Zoology 2020;41(14):9-18.

35. Teronpi V, Singh HT, Tamuli AK, Teron R. Ethnozoology of Karbis of Assam, India: Use of ichthyofauna in traditional health-care practices. Ascient Sci Life 2012;32:99-103.

36. World Health Organization. Fact Sheet No. 134 (December 2008): Traditional Medicine. Geneva, Switzerland 2008.

37. Yirga G, Teferi M, Gebreslassea Y. Ethnozoological study of traditional medicinal animals used by the people of Kafta-Humera District, Northern Ethiopia. Int. J Medicine and Medical Sci 2011;3(10):316-320. 